\title{
Generation of frequency-tunable nanoacoustic waves by optical coherent control
}

\author{
Cheng-Ta Yu, Kung-Hsuan Lin, and Chia-Lung Hsieh \\ Department of Electrical Engineering and Graduate Institute of Electro-Optical Engineering, \\ National Taiwan University, Taipei 10617, Taiwan \\ Chang-Chi Pan and Jen-Inn Chyi \\ Optical Sciences Center, National Central University, Chung-Li 32054, Taiwan \\ Chi-Kuang Sun ${ }^{\text {a) }}$ \\ Department of Electrical Engineering and Graduate Institute of Electro-Optical Engineering, \\ National Taiwan University, Taipei 10617, Taiwan
}

(Received 6 May 2005; accepted 12 July 2005; published online 25 August 2005)

\begin{abstract}
We have developed a system to generate arbitrary wave-form nanoacoustic waves (NAWs) with a piezoelectric InGaN/GaN single-quantum well. Based on an optical coherent control technique, acoustic frequency tunability in the subterahertz range is realized within only one fixed sample. The acoustic generation mechanisms, especially the in-well piezoelectric field Coulomb screening which tends to be saturated at high carrier concentrations, are discussed with optical power dependency. With the generated NAWs propagating in the $c$ axis of a GaN thin film, the lifetime of the $500 \mathrm{GHz}$ longitudinal-acoustic phonon pulses in $\mathrm{GaN}$ is measured to be longer than $420 \mathrm{ps}$, corresponding to a GaN depth more than $3.3 \mu \mathrm{m}$. (C) 2005 American Institute of Physics. [DOI: 10.1063/1.2035882]
\end{abstract}

For recent decades, remarkable advances in femtosecond lasers and ultrafast spectroscopy have enabled investigations of ultrafast phenomena in semiconductors, including coherent phonon processes. The generation and detection of the coherent acoustic phonons have been demonstrated in various semiconductor structures, such as bulk materials, ${ }^{1,2}$ superlattices, ${ }^{3-6}$ quantum dots, ${ }^{7}$ nanoparticles, ${ }^{8}$ and $p-n$ junctions. ${ }^{9}$ In our previous studies, the generation of large amplitude $\mathrm{THz}$ coherent longitudinal-acoustic phonons was demonstrated in piezoelectric heterostructures. ${ }^{10}$ The InGaN multiple-quantum wells (MQWs) containing large in-well piezoelectric fields, treated as optical piezoelectric transducers $(\mathrm{OPT}),{ }^{11,12}$ were utilized to initiate the coherent acoustic waves, and the nanoscaled barrier and well widths of MQWs ensure the extremely short acoustic wavelength. These socalled nanoacoustic waves (NAWs) (Ref. 11) are recently applied to the field of nanoultrasonics ${ }^{12}$ for the usage of nanostructure imaging and THz-bandwidth acoustoelectronic control for nanoscaled devices. ${ }^{13}$

However, because the wavelength of the NAWs generated from the MQW-based OPT is directly determined by the MQW periods, MQWs with different periods are required for various acoustic frequency generation. It is thus not convenient for further frequency-dependent applications. In this letter, we demonstrate an optical coherent control technique with a piezoelectric single-quantum well (SQW) in order to realize tunable acoustic frequency generation. In addition, saturation phenomena of acoustic phonon generation and propagating lifetime of the generated NAWs are also studied.

The experimental setup for optical coherent control is a modification of the typical femtosecond transient transmission pump-probe system. A femtosecond Ti:Sapphire laser, frequency-doubled by a BBO crystal, provides optical pulses with a full width at half maximum pulsewidth of $200 \mathrm{fs}$ at a

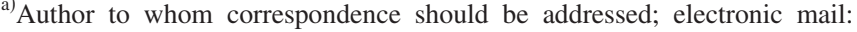
sun@cc.ee.ntu.edu.tw
}

central wavelength of $400 \mathrm{~nm}$. We took the (0001)-oriented InGaN/GaN SQW, which contains high piezoelectric fields on the order of several hundred $\mathrm{kV} / \mathrm{cm}$ inside the nanoscaled quantum well, ${ }^{14}$ as an OPT. Two $\mathrm{In}_{0.2} \mathrm{Ga}_{0.8} \mathrm{~N} / \mathrm{GaN}$ SQW samples, Sample A with a well width of $7 \mathrm{~nm}$ and Sample B with a well width of $2.9 \mathrm{~nm}$, were both grown on $c$-plane sapphire substrates with few-micron-thick unintentionally doped $\mathrm{GaN}$ buffer layers. The $60 \mathrm{~nm}$ thick (for Sample A) and $70 \mathrm{~nm}$ thick (for Sample B) unintentionally doped GaN cap layers grown on the SQW determine how long the generated acoustic waves take to travel from the SQW to the top surface.

Figure 1(a) illustrates the concept of the optical coherent control procedure. An optical pulse train composed of a series of optical pump pulses with fixed time intervals was utilized to excite the carriers in the SQW. The built-in electric field dissociated the photoexcited electrons and holes instantly. ${ }^{15}$ With the piezoelectric field partially screened, the strain redistribution initiated a series of acoustic pulses, assembling NAWs due to the nanoscaled pulsewidths determined by the well thickness. By adjusting the optical time delays between the pump pulses, the acoustic frequency can be easily tuned in the subterahertz range.

The strained SQW, treated as an acoustic waveform synthesizer, cannot only generate NAWs but also be utilized for high-bandwidth acoustic detection. ${ }^{16}$ The strain waves modified the piezoelectric field while propagating through the well region. Therefore, the optical absorption of the SQW was modulated due to the quantum confined Franz-Keldysh (Ref. 17) effect and the transient transmission measurement of a femtosecond optical probe pulse can clearly monitor the transient strain distribution of the NAWs once they propagated through the SQW. In our experiments, the NAW generated from the InGaN SQW propagated in opposite directions: As shown in Fig. 1(a), one propagated into the cap layer and was reflected from the surface; the other propagated into the GaN buffer layer that is much thicker than the 


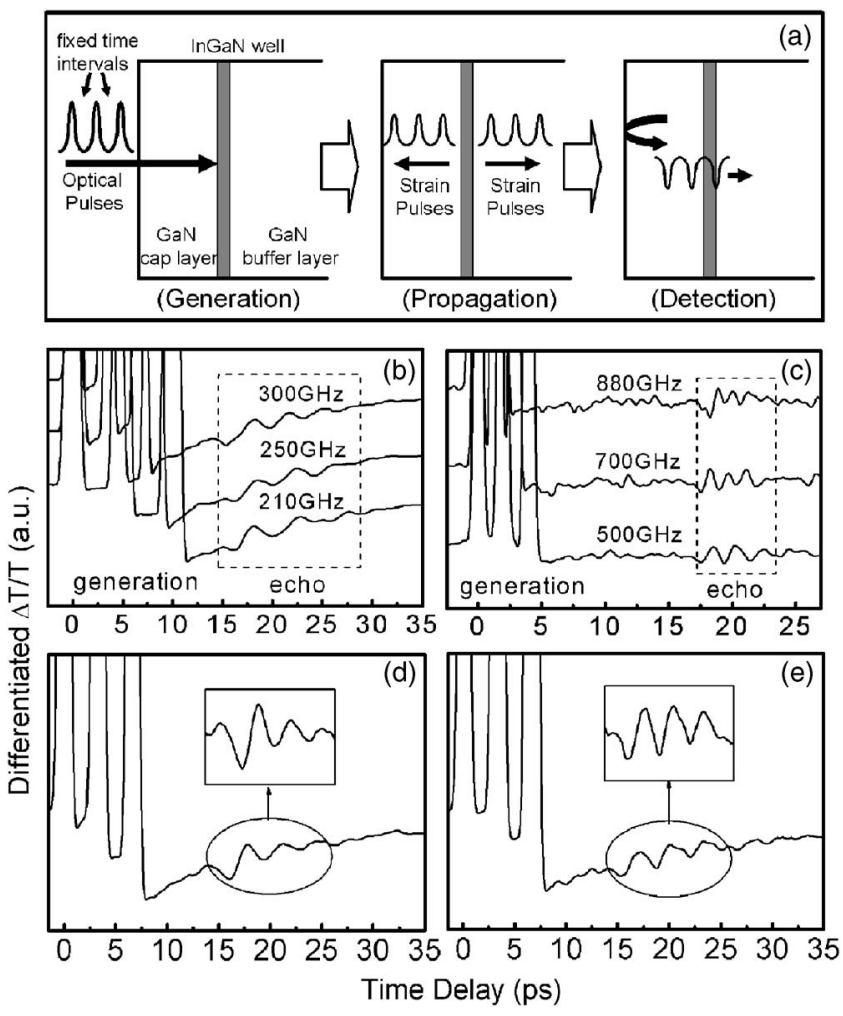

FIG. 1. (a) A schematic diagram illustrating the nanoacoustic-wave generation. The echo of the generated acoustic pulse propagating into the cap layer will be detected by the SQW. (b,c) Differentiated transient transmission measurement demonstrates the optical coherent control of the generated acoustic frequency in the (b) $7 \mathrm{~nm}$ thick and (c) $2.9 \mathrm{~nm}$ thick InGaN SQW. $(\mathrm{d}, \mathrm{e})$ NAWs initiated by three optical pumps (d) with equivalent power (16 $\mathrm{mW}, 16 \mathrm{~mW}, 16 \mathrm{~mW}$ ) and (e) after raising the power of the second and third pumps $(10 \mathrm{~mW}, 16 \mathrm{~mW}, 19 \mathrm{~mW})$.

cap layer and then went straight into the sapphire substrate. In this letter, we focus our discussion on the detection of the acoustic echo reflected from the surface.

As shown in Figs. 1(b) and 1(c), we demonstrate the optical coherent control with three optical pump pulses. The NAWs whose frequencies ranged between $100 \mathrm{GHz}$ and 880 $\mathrm{GHz}$ were generated and detected in Sample A (up to 500 $\mathrm{GHz}$ ) and Sample B (up to $880 \mathrm{GHz}$ ). The upper limit of the generated NAW frequency was restricted by the well thickness and the optical pulse width. With Sample A, as shown in Fig. 1(b), the generated acoustic frequency was upperlimited to be $0.5 \mathrm{THz}$ due to the $7 \mathrm{~nm}$ thick well, corresponding to a $\sim 1$ ps acoustic traveling time (the sound velocity in $c$ axis of $\mathrm{GaN}$ is about $8 \mathrm{~nm} / \mathrm{ps}),{ }^{9}$ and Fig. 1(c) shows that we can generate higher acoustic frequency up to nearly 1 $\mathrm{THz}$ with Sample B containing a much thinner SQW. It is apparently possible for us to obtain NAWs with various frequencies in just one SQW sample.

When utilizing the optical coherent control, a piezoelectric field saturation effect was observed. With three optical pumps of equally $16 \mathrm{~mW}$ average power initiating the acoustic pulses, as shown in Fig. 1(d), it is obvious that the second and third generated acoustic oscillations were weaker than the first one, which indicates a saturation phenomenon of the acoustic generation. Under our experimental condition, the optical absorbed power in the SQW was confirmed to be linear with the optical pump power, which indicates that the total power of the three optical pump pulses did not saturate the carrier excitation in the SQW. Therefore, the observed Downloaded 16 Feb 2009 to 140.112 .113 .225 . Redistribution subje

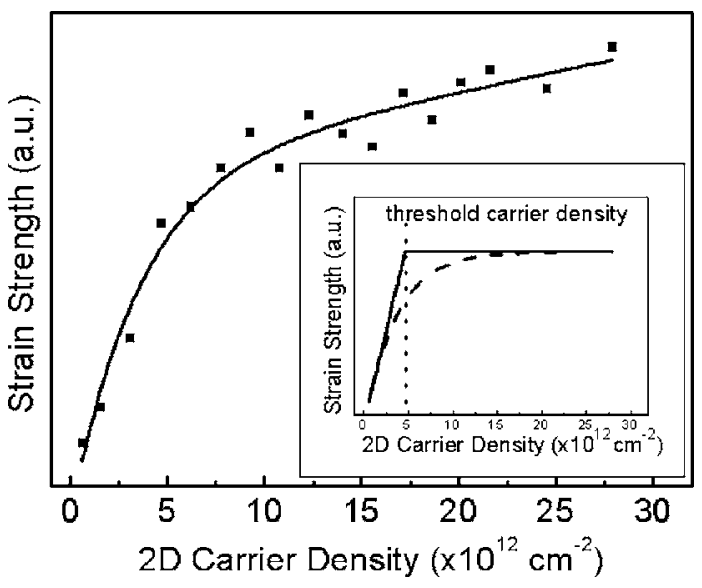

FIG. 2. The relative strain strength obtained from differentiated transient reflectivity measurement versus the 2D carrier density in the SQW (solid square). The fitting curve is represented by the solid line. The dashed line in the inset shows the saturation term extracted from the fitting curve and the solid line is depicted according to the charged-parallel-plate approximation. A threshold carrier density of $3.6 \times 10^{12} \mathrm{~cm}^{-2}$ is marked by the dotted line.

saturation phenomenon should be strongly correlated with the most important generation mechanism, that is, the screening efficiency of the in-well piezoelectric field.

To further investigate the physical mechanism, we have also performed a transient reflectivity pump-probe measurement. $^{2,18}$ From the simple relations of $\delta n(z, t)$ $=(\partial n / \partial \eta) \times \eta(z, t)$ and $|\Delta R(t) / R|_{t=t_{0}} \propto|\delta n|$, where $\delta n$ is the refractive index change resulting from the propagating strain pulses along the $z$ direction in transmission materials, ${ }^{2,18}$ one can relate the transient reflectivity change $\Delta R / R$ of the optical probe signal linearly to the strain field $\eta$ which indicates the strength of the generated acoustic pulse. Figure 2 shows the measured strain strength as a function of twodimensional (2D) photoexcited carrier density for Sample A. The 2D carrier density in SQW was obtained by the experimentally measured optical absorbed power with a focused laser beam radius of $8 \mu \mathrm{m}$. The estimated value was further carefully calibrated by comparing with the absorption in a 3 period InGaN/GaN MQW sample in order to remove the influence of buffer layers. As shown in Fig. 2, the acoustic pulse generation was gradually saturated with increased carrier density. This saturation effect is attributed to the saturation of the field screening effect. The slower strain increase after saturation can be attributed to other acoustic generation mechanisms such as deformation potential coupling. ${ }^{19-21} \mathrm{We}$ used two components to fit the experimental data in Fig. 2, one saturation component (as shown in the inset) and a slower linear increase component.

From the exponentially fitted curve corresponding to the saturation term (depicted by the dashed line in the inset of Fig. 2), we defined the threshold carrier density according to the exponential decay factor. We can then estimate the strength of the built-in piezoelectric field inside the SQW with the extracted 2D threshold carrier density and a simple parallel-plate-capacitor model (solid line of the inset of Fig. 2 ), by assuming the photoexcited carriers as $2 \mathrm{D}$ sheet charge carriers distributing on opposite sides of the SQW. Thus, the estimated piezoelectric field was found to be $\sim 1.1 \mathrm{MV} / \mathrm{cm}$, which is in excellent agreement with the reported value, ${ }^{22}$ further supporting our hypothesis.

to AIP license or copyright; see http://apl.aip.org/apl/copyright.jsp 


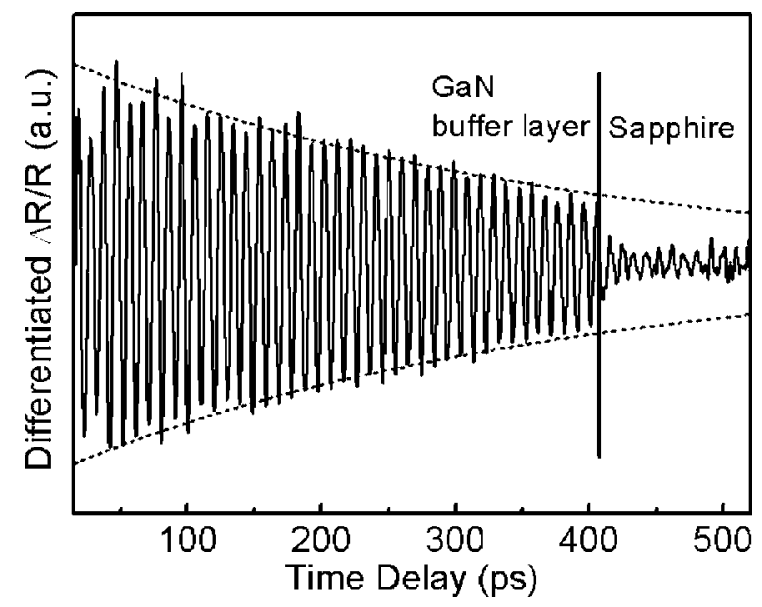

FIG. 3. Differentiated transient reflection measurement of sample A with $500 \mathrm{GHz}$ acoustic waves propagating in the GaN. An attenuating envelope is shown and an acoustic effective lifetime of 420 ps is estimated.

From Fig. 2, the carrier-screening effect obviously dominates the acoustic generation mechanisms before the in-well piezoelectric field is mostly screened. Therefore, it is important to engineer the intensities of the pulse sequence to accurately control the generated acoustic waveform in order to take advantage of the high acoustic generation efficiency of the piezoelectric field screening effect. In Fig. 1(e), we raised the power of the second and third optical pumps to compensate the saturation phenomenon, and a NAW with almost equivalent oscillation amplitudes can then be generated.

We have also utilized the transient reflectivity pumpprobe measurement to measure the acoustic lifetime. As in previous discussions, the reflected probe change modulated by the propagating acoustic wave indicates the acoustic strength. With a generated NAW with a frequency of 500 $\mathrm{GHz}$, Fig. 3 shows the measured transient reflectivity, which is dominated by the dynamic Fabry-Perot effect due to the traveling of the generated NAW in the sample., ${ }^{218}$ After taking the material loss of the optical probe into consideration, ${ }^{23}$ we can then estimate the acoustic lifetime of the generated NAW propagating in the $c$ axis of the GaN substrate. From Fig. 3, the acoustic effective lifetime (including the scattering loss) of a $500 \mathrm{GHz}$ NAW pulse can be estimated to be $\sim 420 \mathrm{ps}$ in GaN, corresponding to a skin depth of approximately $3.3 \mu \mathrm{m}$. It is obvious that the signal becomes very noisy at around 406 ps because of the acoustic wave propagating into the sapphire substrate.

In summary, we have demonstrated an optical coherent control technique to generate NAWs with tunable acoustic frequencies in the subterahertz range. Sequentially excited photocarriers initiate NAWs from a piezoelectric SQW. The acoustic generation efficiency is strongly related to the carrier screening effect of the in-well piezoelectric field, which tends to be saturated with increasing photoexcited carriers. For the generated NAW with a $500 \mathrm{GHz}$ central frequency, a phonon lifetime longer than 420 ps can be resolved, corresponding to a skin depth of $\sim 3.3 \mu \mathrm{m}$ in $\mathrm{GaN}$. As a source of frequency-tunable NAWs, this demonstrated optical coherent control system provides a tunable option for future nanoacoustic applications, for example, highresolution ultrasonics.

This work was sponsored by the National Science Council of Taiwan, R.O.C. under Grant No. 93-2120-M-002-004 and No. 93-2215-E-008-005.

${ }^{1}$ C. Thomsen, J. Strait, Z. Vardeny, H. J. Maris, J. Tauc, and J. J. Hauser, Phys. Rev. Lett. 53, 989 (1984).

${ }^{2}$ C. Thomsen, H. T. Grahn, H. J. Maris, and J. Tauc, Phys. Rev. B 34, 4129 (1986).

${ }^{3}$ A. Yamamoto, T. Mishina, Y. Masumoto, and M. Nakayama, Phys. Rev. Lett. 73, 740 (1994).

${ }^{4}$ A. Bartels, T. Dekorsy, H. Kurz, and K. Köhler, Phys. Rev. Lett. 82, 1044 (1999).

${ }^{5}$ N.-W. Pu and J. Bokor, Phys. Rev. Lett. 91, 076101 (2003).

${ }^{6}$ J. J. Baumberg, D. A. Williams, and K. Köhler, Phys. Rev. Lett. 78, 3358 (1997).

${ }^{7}$ T. D. Krauss and F. W. Wise, Phys. Rev. Lett. 79, 5102 (1997).

${ }^{8}$ M. Nisoli, S. De Silvestri, A. Cavalleri, A. M. Malvezzi, A. Stella, G. Lanzani, P. Cheyssac, and R. Kofman, Phys. Rev. B 55, 13424 (1997).

${ }^{9}$ K.-H. Lin, C.-T. Yu, Y.-C. Wen, and C.-K. Sun, Appl. Phys. Lett. 86, 093110 (2005).

${ }^{10}$ C.-K. Sun, J.-C. Liang, and X.-Y. Yu, Phys. Rev. Lett. 84, 179 (2000).

${ }^{11}$ K.-H. Lin, C.-L. Hsieh, T.-M. Liu, S. Keller, S. P. DenBaars, G.-T. Chen, C.-C. Pan, J.-I. Chyi, and C.-K. Sun, Proc. SPIE 5352, 101 (2004).

${ }^{12}$ K.-H. Lin, G.-W. Chern, C.-T. Yu, T.-M. Liu, C.-C. Pan, G.-T. Chen, J.-I. Chyi, S.-W. Huang, P.-C. Li, and C.-K. Sun, IEEE Trans. Ultrason. Ferroelectr. Freq. Control (to be published).

${ }^{13}$ K.-H. Lin, G.-W. Chern, Y.-K. Huang, and C.-K. Sun, Phys. Rev. B 70, 073307 (2004).

${ }^{14}$ S. F. Chichibu, A. C. Abare, M. P. Mack, M. S. Minsky, T. Deguchi, D. Cohen, P. Kozodoy, S. B. Fleischer, S. Keller, J. S. Speck, J. E. Bowers, E. Hu, U. K. Mishra, L. A. Coldren, S. P. DenBaars, K. Wada, T. Sota, and S. Nakamura, Mater. Sci. Eng., B 59, 298 (1999).

${ }^{15}$ A. Shikanai, T. Deguchi, T. Sota, T. Kuroda, A. Takeuchi, S. Chichibu, and S. Nakamura, Appl. Phys. Lett. 76, 454 (2000).

${ }^{16}$ G.-W. Chern, C.-K. Sun, G. D. Sanders, and C. J. Stanton, Top. Appl. Phys. 92, 339 (2004).

${ }^{17}$ D. A. B. Miller, D. S. Chemla, and S. Schmitt-Rink, Phys. Rev. B 33, 6976 (1986)

${ }^{18}$ R. Liu, C. S. Kim, G. D. Sanders, C. J. Stanton, J. S. Yahng, Y. D. Jho, K. J. Yee, E. Oh, and D. S. Kim, cond-mat/0310654.

${ }^{19}$ G. D. Sanders and C. J. Stanton, and C. S. Kim, Phys. Rev. B 64, 235316 (2001).

${ }^{20}$ G. D. Sanders and C. J. Stanton, and C. S. Kim, Phys. Rev. B 66, 079903 (2002).

${ }^{21}$ F. Chen, M. C. Cheung, P. M. Sweeney, W. D. Kirkey, M. Furis, and A. N. Cartwrighta, J. Appl. Phys. 93, 4933 (2003).

${ }^{22}$ C. Y. Lai, T. M. Hsu, W.-H. Chang, K.-U. Tseng, C.-M. Lee, C.-C. Chuo, and J.-I. Chyi, J. Appl. Phys. 91, 531 (2002).

${ }^{23}$ O. Ambacher, W. Rieger, P. Ansmann, H. Angerer, T. D. Moustakas, and M. Stutzmann, Solid State Commun. 97, 365 (1996). 